\title{
MOLECULAR RESONANCES OBSERVED IN THE PREDISSOCIATION OF Cs2
}

\author{
BONGSOO KIM ${ }^{\mathrm{a}}$ and KEITARO YOSHIHARA \\ Institute for Molecular Science, Myodaiji, Okazaki 444, Japan
}

(Received 30 July, 1994)

\begin{abstract}
Very cold molecular beam of $\mathrm{Cs}_{2}$ is generated by a high temperature supersonic jet source. State-specific photofragment yield spectrum is obtained in the orange band of $\mathrm{Cs}_{2}$. Asymmetric line shapes are observed. The Fano line shape parameter, $q$, shows a gradual sign change, which is called " $q$-reversal". A very broad absorption band which has about $50 \mathrm{~cm}^{-1}$ width is observed and explained to occur through the perturbation by the $D^{1} \Sigma_{u}^{+}$state. The complex resonance line shapes are attributed to the interfering resonances.
\end{abstract}

KEY WORDS: Cesium dimer, Predissociation, Complex resonance, q-reversal

\section{INTRODUCTION}

In the last few years, we have seen increasing interest in a new field called 'transition state spectroscopy', in which spectroscopic methods are used to probe directly the transition region of molecules where chemical bonds are about to form or break. ${ }^{1,2}$ This 'transition-region species', which refers to the short-lived molecular complex in this unstable region, is a quasibound state occurring when discrete states are coupled to a continuum, and can be called 'resonance' in more general term. In the study of molecular reaction dynamics, the resonance can be a very sensitive probe of the reactive scattering processes, providing spectroscopic information on the detailed process of chemical changes. ${ }^{3}$

The resonance occurring in the predissociation of diatomic molecules is the simplest example of such transition-region species. There are close similarities between the diatomic predissociation and the atomic autoionization, with the fragmented particle being an atom in the former, and an electron in the latter. The resonances in the autoionization have been studied in much more detail than in the predissociation. Fano showed that lineshapes in the autoionization can be described by a simple formula when a discrete state in coupled to a single continuum, as

\footnotetext{
a Permanent address: Department of Chemistry Education, Kyungpook National University, Taeku 702-701, Korea
} 


$$
\sigma_{a}=\sigma_{0} \frac{(\varepsilon+q)^{2}}{1+\varepsilon^{2}}
$$

where $\varepsilon=\left(E-E_{r}\right) /(\Gamma / 2)$ is the reduced energy, $\Gamma$ is the width of the resonance, $E_{r}$ is the resonance energy, $\sigma_{0}$ corresponds to the dissociative continuum cross section, and $q$ is the line shape parameter. ${ }^{4}$

Among alkali dimers, $\mathrm{Cs}_{2}$ shows the most complex spectrum due to very compact potential energy curves and large spin-orbit couplings. They are, therefore, very suitable to study the predissociation occurring through multichannel interactions. When illuminated by blue or orange light, $\mathrm{Cs}_{2}$ is known to produce excited Cs atoms in several different internal states. ${ }^{5}$ Detailed spectroscopic studies on the photodissociation of $\mathrm{Cs}_{2}$ have, however, been hampered by the complex absorption spectrum caused by numerous hot bands and its very small rotational constant. We have designed a new high temperature pulsed nozzle and prepared a very cold molecular beam of $\mathrm{Cs}_{2}$, in which all the vibrational hot bands are practically eliminated and the rotational temperature is reduced to about $1 \mathrm{~K} \cdot{ }^{6-12}$ In this very cold molecular beam the number of partial cross sections for each $\mathrm{J}$, the angular momentum of the initial state, superimposed in the observed dissociation cross section, are greatly reduced. The dramatic cooling in a pulsed molecular beam turns out to be very important in the study of resonance, since direct comparison with theory now becomes possible.

\section{EXPERIMENTAL}

Figure 1 shows the experimental setup. $\mathrm{Cs}_{2}$ is produced by expanding $\mathrm{Cs}$ vapor with $\mathrm{Kr}$ gas using a high temperature pulsed nozzle. The nozzle is made of a modified fuel injector of an automobile engine (Nippon Denso). An electric pulse of $1 \mathrm{msec}$ duration and $50 \mathrm{~V}$ height is applied to open the nozzle. The diameter of the nozzle is $0.8 \mathrm{~mm}$. While the pulsed nozzle set is heated to about $450^{\circ} \mathrm{C}$, the electromagnet part is cooled down to room temperature by water. We fill 1 gram of $99.95 \%$ Cs (Mitsuwa Chemicals) in the sample room of the pulsed nozzle, which lasted for longer than one week when the nozzle was operated continuously at $4 \mathrm{~Hz}$. The most difficult part of the experiment is keeping the nozzle in continuous operation in order to prevent the condensation of the Cs, which hinders smooth operation of the pulsed beam. The pulsed supersonic jet, in which $\mathrm{Cs}, \mathrm{Cs}_{2}$, and the seeding gas $(\mathrm{Kr})$ were the major species, was collimated by a $1.2 \mathrm{~mm}$ diameter skimmer (Beam Dynamics) located $5 \mathrm{~cm}$ from the nozzle. The skimmed molecular beam was intersected at right angles $15 \mathrm{~cm}$ from the skimmer by the pump and probe laser pulses that were propagating collinearly. A typical pressure was $2 \times 10^{-4}$ Torr for the source chamber and $4 \times 10^{-7}$ Torr for the detection chamber when the pulsed nozzle was operated at $4 \mathrm{~Hz}$ with 600 Torr of $\mathrm{Kr}$ backing pressure.

Ions formed by the absorption of two photons from the excitation laser were accelerated by a double electrostatic field time-of-flight lens to about $3500 \mathrm{eV}$ and traveled through a $70 \mathrm{~cm}$ long field free region toward a dual microchannel plate detector. The ion signals were then amplified by a fast preamplifier and sent to 


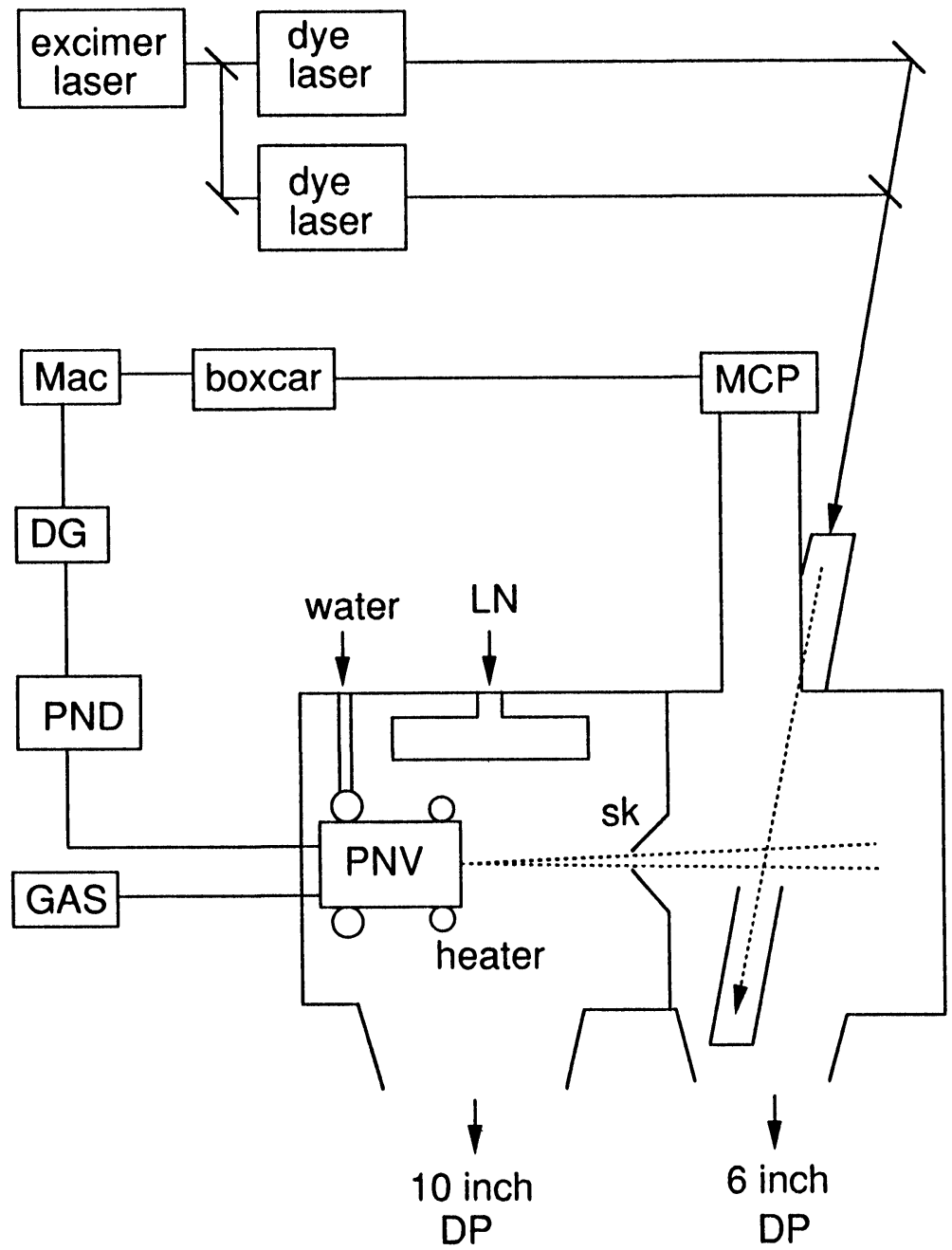

Figure 1 Schematic illustration of the molecular beam apparatus with the linear time-of-flight mass spectrometer. DG: delay generator, PND: pulsed nozzle driver, PNV: pulsed nozzle valve, SK: skimmer, MCP: microchannel plate detector, LN: liquid nitrogen, GAS: gas supply system.

boxcar integrators. By collimating the laser beam with a skimmer, we could achieve very good mass selection, with the mass resolution of about 500 at the mass of ${ }^{133} \mathrm{Cs}_{2}$ (265.8 a.u.). The ion signals were monitored by using two boxcar integrators. By adjusting the time gates of the two boxcars, $\mathrm{Cs}^{+}$and $\mathrm{Cs}_{2}^{+}$ion signals were both recorded at the same time. The frequency of the excitation laser was measured simultaneously at the time of the experiment by sending a fraction of the laser beam through an optogalvanic device filled with Ne. The pump and probe laser pulses were provided by two dye lasers pumped by an excimer laser. The probe laser pulse was delayed 5 nanosecond with respect to the pump pulse by optical path length 
difference. The nominal bandwidth of the pump and probe laser pulses are $0.2 \mathrm{~cm}^{-1}$. The pump and probe laser power were reduced to less than $200 \mu \mathrm{J}$ per pulses by neutral density filters in order to avoid saturation and power broadening.

\section{RESULTS AND DISCUSSION}

Figure 2 shows the observed ionization spectra obtained in the supersonic molecular beam of $\mathrm{Cs}_{2}$. The upper trace is the resonance enhanced two photon ionization spectrum of $\mathrm{Cs}_{2}$, in which $\mathrm{Cs}_{2}^{+}$ions are detected. The lower trace is the photofragment yield (PFY) spectrum of $\mathrm{Cs}_{2}$, in which Cs $\left(6{ }^{2} \mathrm{P}_{3 / 2}\right)$ atom resulted from the photodissociation of $\mathrm{Cs}_{2}$ is selectivity monitored by a single photon ionization. The ionization laser has an energy of $19720 \mathrm{~cm}^{-1}$, which is larger than the ionization energy for the Cs $\left(6{ }^{2} \mathrm{P}_{3 / 2}\right)$ atom $\left(19674.36 \mathrm{~cm}^{-1}\right)$. Extra bands observed in the PFY spectrum at frequencies higher than $17100 \mathrm{~cm}^{-1}$ are due to the $\mathrm{Cs}^{+}$ions fragmented from the $\mathrm{Cs}_{2}^{+}$ ions generated by two photon ionization and correspond to the vibrational progression of the $D^{1} \Sigma_{u}^{+}$state. ${ }^{18}$ When the energy of the ionization laser (probe pulse) is less than the ionization energy $\left(19674.36 \mathrm{~cm}^{-1}\right)$, all the peaks except those extra bands disappear. The rotational temperature of $\mathrm{Cs}_{2}$ in the molecular beam in estimated to be about $1.0 \mathrm{~K}$ by simulating the rotational contours with the known molecular parameters of the D and X states. The intensity of the hot bands are very weak, and the vibrational temperature is thus estimated to be lower than $5 \mathrm{~K}$.

Figure 3 shows the ionization schemes of these spectra. The first laser prepares $\mathrm{Cs}_{2}$ molecule in its excited state. The coupling to the repulsive electronic state predissociates the excited $\mathrm{Cs}_{2}$ molecule. The second laser pulse of frequency $v_{2}$ ionizes the dissociated Cs $\left(6{ }^{2} \mathrm{P}_{3 / 2}\right)$ atom. When the second laser pulse of frequency $v_{2}$ is applied to the excited $\mathrm{Cs}_{2}$ molecule, two electronic processes are in competition. They are the predissociation caused by the coupling of the excited bound state to the continuum and the molecular ionization caused by the absorption of the second photon of the frequency of either $v_{1}$ or $v_{2}$. If the predissociation rate is fast, or in the order of picosecond, the molecular ionization process becomes much less effective, making the $\mathrm{Cs}_{2}^{+}$ion signal too weak to observe. When the predissociation rate is in the order of nanosecond, we observe both the predissociated atomic fragments $\left(\mathrm{Cs}^{+}\right)$and the $\mathrm{Cs}_{2}^{+}$ions.

The experimental PFY spectrum in Fig. 2 (lower trace) shows many broad and asymmetric line profiles. At close inspection, the PFY spectrum shows a gradual sign change of the line shape parameter $q$ passing through zero (near $16900 \mathrm{~cm}^{-1}$ ) and through infinity (near $17050 \mathrm{~cm}^{-1}$ ). The Beutler-Fano profile remains valid only in the isolated resonance case, i.e., when only a single level of the discrete state is coupled to the continuum. When more than a single discrete state are coupled to the continuum, second and higher order perturbations should be taken into account, and the profile cannot be described by the simple Fano formula. Note that the band near $17050 \mathrm{~cm}^{-1}$ cannot be simulated by a single Beutler-Fano profile and shows clear multichannel quantum interference effect. 

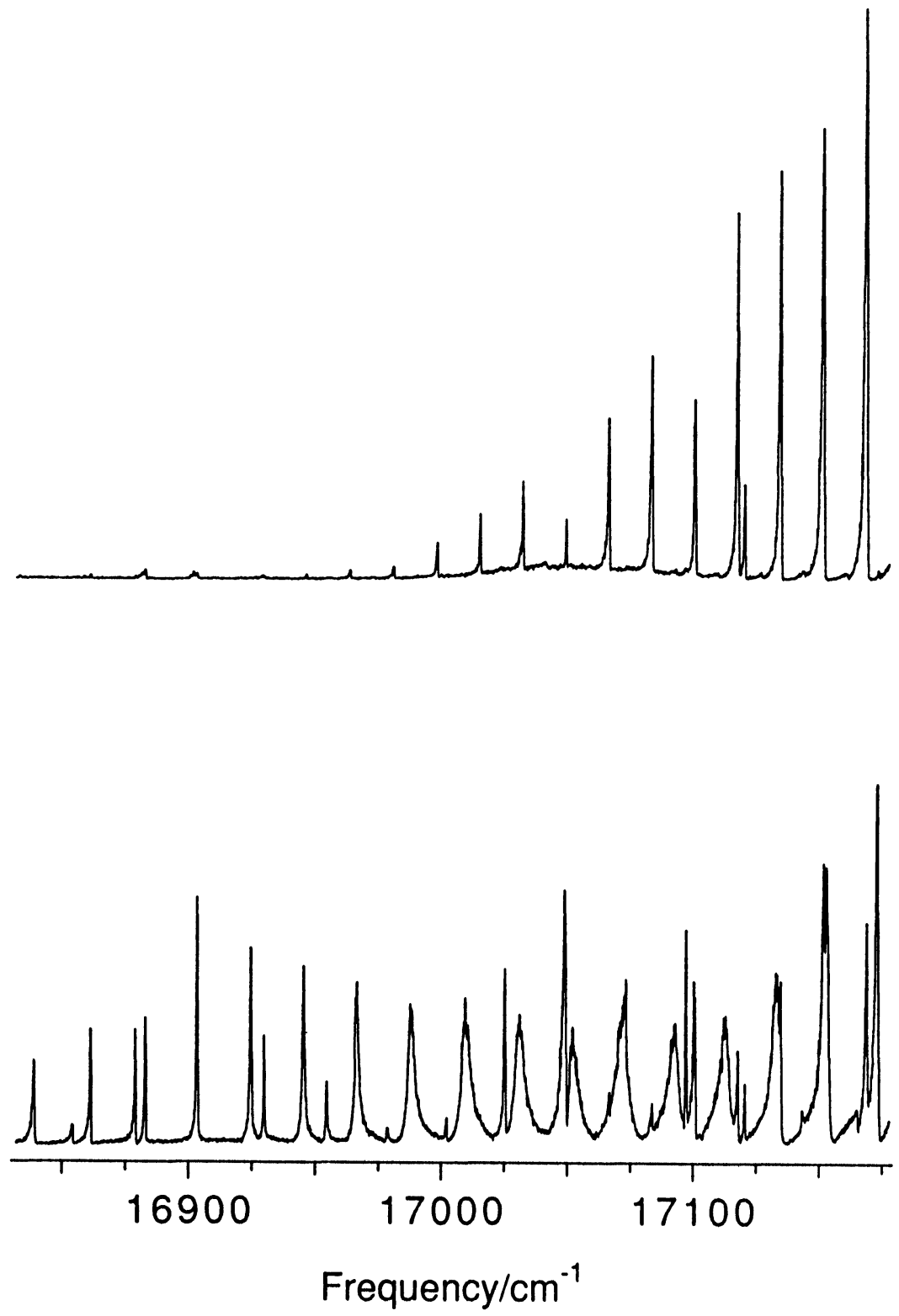

Figure 2 Ionization spectrum of $\mathrm{Cs}_{2}$. The seeding gas is $1 \mathrm{~atm}$. of $\mathrm{Kr}$. a) Upper trace: 2 photon ionization spectrum of $\mathrm{Cs}_{2}$. Cs $s_{2}^{+}$ion is monitored. b) Lower trace: photofragment yield (PFY) spectrum of $\mathrm{Cs}_{2}$. Cs $\left(6{ }^{2} \mathrm{P}_{3 / 2}\right)$ atom is ionized and monitored.

The possibility that the asymmetric line shapes are due to rotational structures is excluded based on the following evidences. Firstly, the vibrational progressions show very regular spacing. Rotational bands for diatomic molecules are asymmetric and blue-shaded if the rotational constant of the excited state $\left(\mathrm{B}^{\prime}\right)$ is larger than that of 
molecular

ionization

continuum

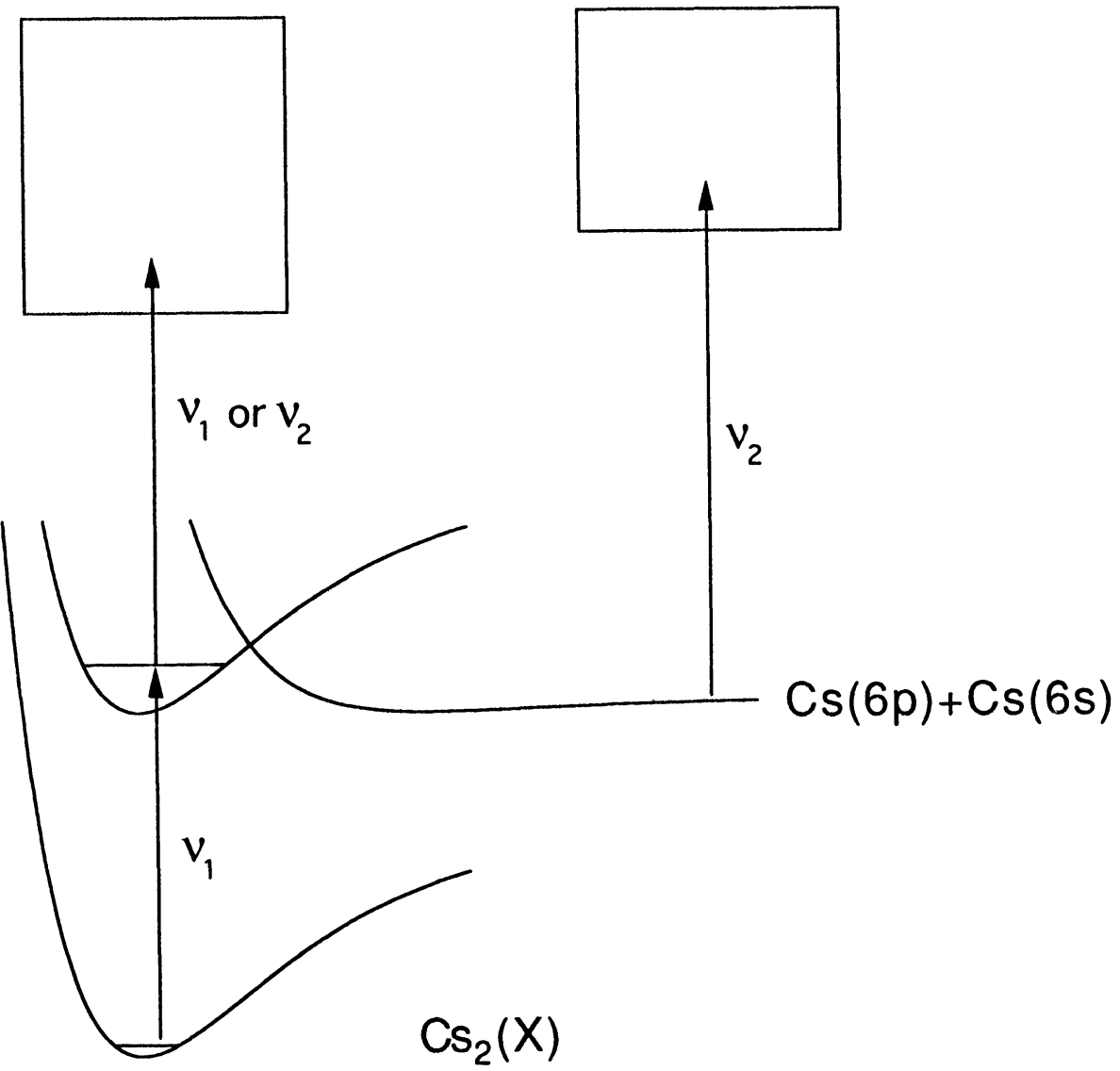

atomic

ionization

continuum

Figure 3 The ionization scheme of the spectra shown in Fig. 2.

the ground state $\left(\mathrm{B}^{\prime \prime}\right)$, and red-shaded if $\mathrm{B}^{\prime}$ is smaller than $\mathrm{B}^{\prime \prime}$. The rotational constant $\left(B^{\prime}\right)$, however, would not change so widely in a region where the potential curve is close to harmonic. Secondly, if the broadening were due to rotational structures, its shape would change when the rotational temperature of the $\mathrm{Cs}_{2}$ in molecular beam is changed. Figure 4 shows the ionization spectrum of the $\mathrm{Cs}_{2}^{+}$(upper trace) and $\mathrm{Cs}^{+}$ ions (lower trace) with the coexpanding gas changed to $\mathrm{Ar}$ and backing pressure reduced to 100 Torr. Under these expansion conditions, the rotational temperature becomes higher than $5 \mathrm{~K}$. In the spectra of Fig. 4, however, the line shapes of the broad resonances of the $\mathrm{Cs}^{+}$signal remain about the same. This observation shows that the broad and asymmetric line shapes are not due to rotational structures. Lastly, the band at $17050 \mathrm{~cm}^{-1}$ shows a dip in the center, which cannot be explained by a rotational structure. 

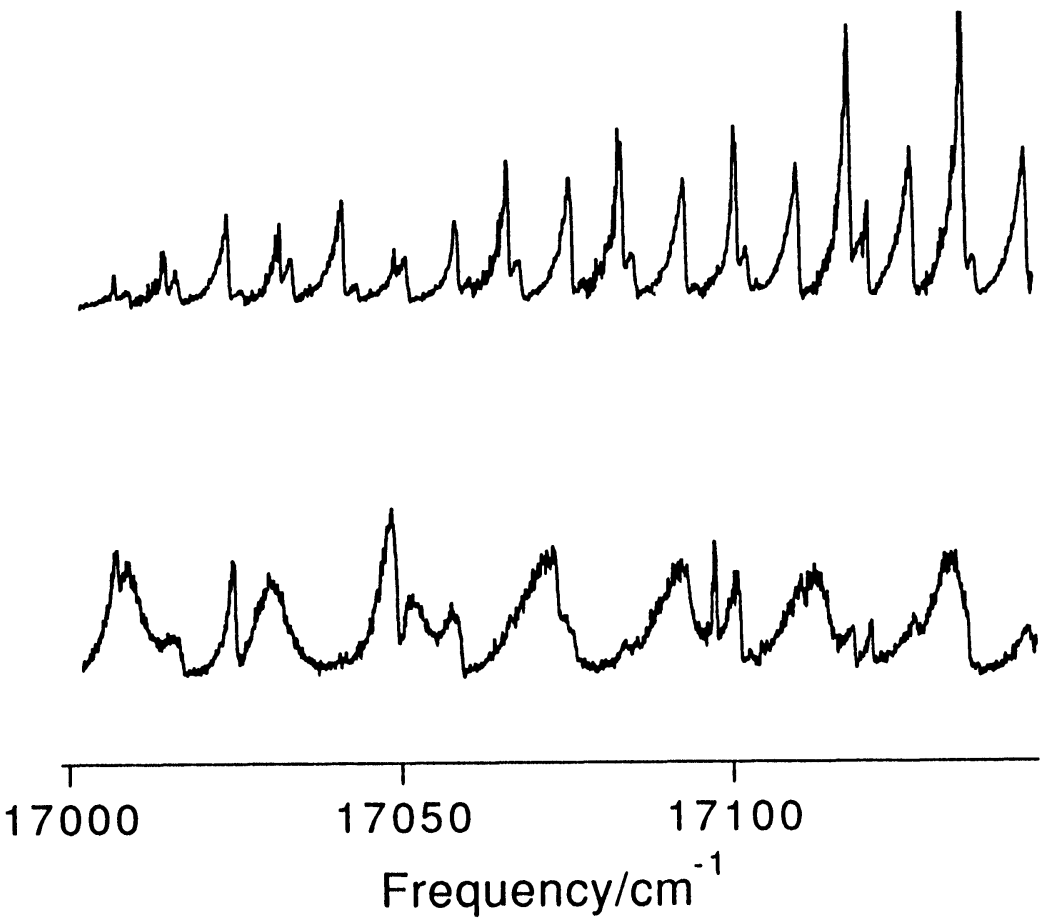

Figure 4 Ionization spectra of $\mathrm{Cs}_{2}$ obtained under the same conditions as in Fig. 2, except that the seeding gas in changed to 100 torr of Ar. The line shape of the broad resonances are not changed.

Figure 5 shows the potential energy diagram of $\mathrm{Cs}_{2}$ in the interested frequency range. The potential curves are from the $a b$ initio calculations by Spiess and Meyer obtained by the pseudo-potential method..$^{13}$ The spectroscopic data on these electronic states have been fragmentary. Only the low vibrational levels of the $C^{1} \Pi_{u}$ (up to $\mathrm{v}^{\prime}=13$ ), ${ }^{14}$ and (2) ${ }^{3} \Pi_{u}$ states (up to $\mathrm{v}^{\prime}=6$ ) $^{8}$ were observed previously. Complex perturbations are observed for higher vibrational levels of the $C^{1} \Pi_{u}$ state. The $\Omega=0$ component of the (2) ${ }^{3} \Pi_{u}$ state is predicted to perturb the $D^{1} \Sigma_{u}^{+}$state at frequencies higher than $17500 \mathrm{~cm}^{-1}$, which was confirmed recently. ${ }^{15}$

Among these three electronic states, only the $C^{1} \Pi_{u}$ state has oscillator strength associated with the ground state, which has a ${ }^{1} \Sigma_{g}^{+}$symmetry. Since the equilibrium bond lengths of the $C^{1} \Pi_{u}$ and (2) ${ }^{3} \Pi_{u}$ states are very close to that of the ground state, the high vibrational levels of the $C^{1} \Pi_{u}$ and (2) ${ }^{3} \Pi_{u}$ states have very small Franck-Condon overlap with the $\mathrm{v}^{\prime \prime}=0$ level of the ground state at the observed frequency range. Only the (2) ${ }^{3} \Sigma_{u}^{+}$state, which is a continuum state, has a good Franck-Condon overlap with the ground state $\left(v^{\prime \prime}=0\right)$ at the observed frequency range, and carries most of the oscillator strength, which is borrowed from the low vibrational levels $(\mathrm{v}=0 \sim 6)$ of the $C^{1} \Pi_{u}$ state. The resonances occurring in this system are then described as a result of configuration interactions between dark discrete states near the resonance energy, a dark continuum, and remote bright discrete states. 


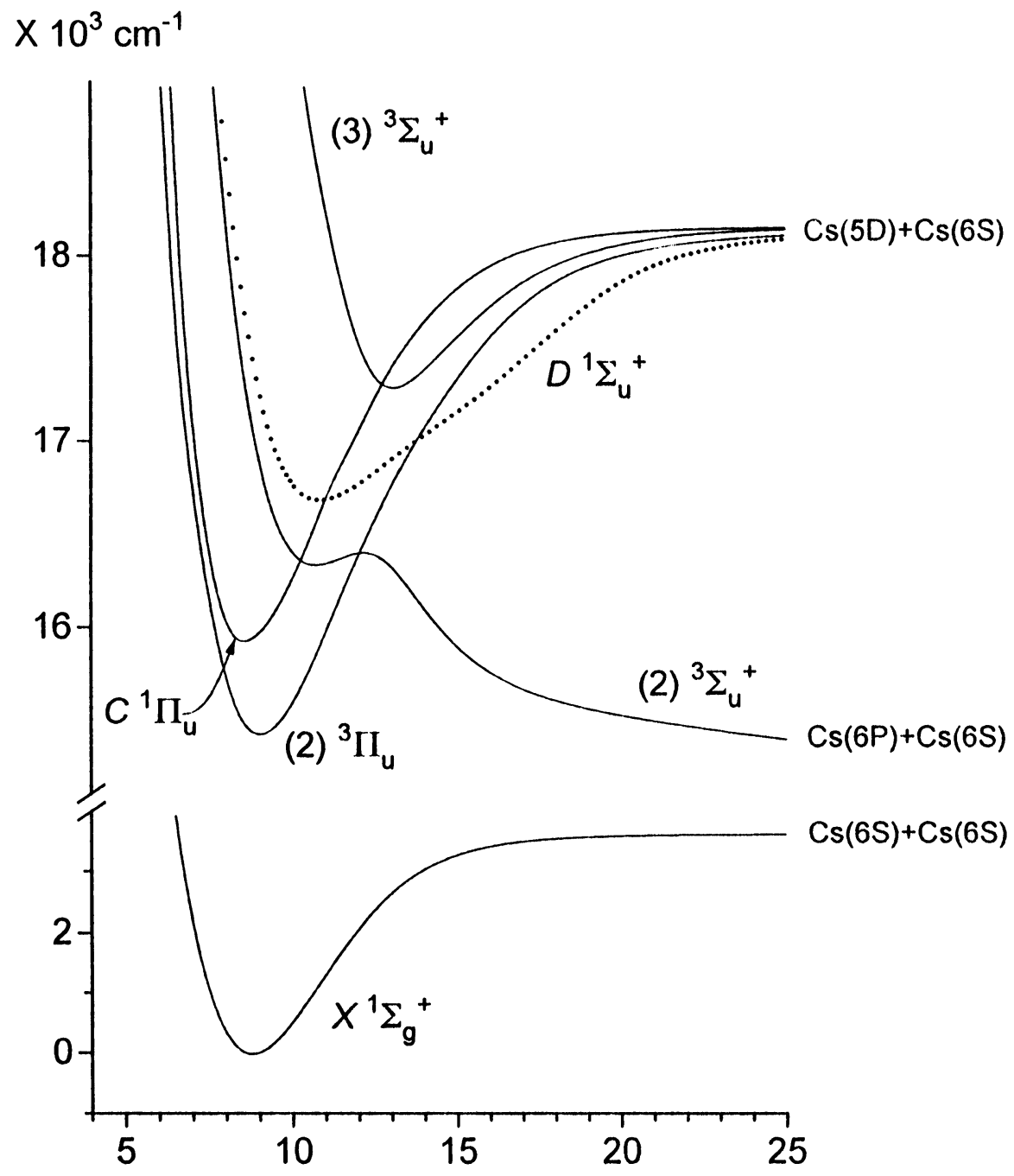

INTERNUCLEAR DISTANCE (Bohr)

Figure 5 The potential energy curves of $\mathrm{Cs}_{2}$, obtained by ab initio calculations.

The PFY spectrum in Fig. 2 shows a couple of very narrow resonance lines (at 17097 and $17020 \mathrm{~cm}^{-1}$ ). The narrow resonances with a very long lifetime have been observed in the autoionization of $\mathrm{Ba}$ atom and that of $\mathrm{Li}$ atom in strong magnetic field. ${ }^{16,17}$ They are interpreted as occurring when several resonances associated with different channels interfere strongly and the coupling matrix elements to the open channel are accidentally canceled out. This produces a metastable state with an anomalously long lifetime, which is referred to as a 'bound state in a continuum' 
(BIC). The close-coupling calculations predict the existence of several very narrow resonances. ${ }^{18}$ Since this resonance is borrowing the oscillator strength from the continuum of (2) ${ }^{3} \Sigma_{u}^{+}$, which borrows the oscillator strength from the low vibrational levels of the $C^{1} \Pi_{u}$ state in turn, the radiative lifetime is much longer than that of the low vibrational levels of the $C^{1} \Pi_{u}$ state and would be of the same order as the predissociation lifetime.

Compared to some broad resonances as wide as about $5 \mathrm{~cm}^{-1}$ (fwhm) and thus about $600 \mathrm{fs}$ of lifetime, these narrow resonances are expected to have lifetimes in the order of nanoseconds, which is more than about $10^{4}$ times enhancement of the lifetime. While the BIC's in the autoionization can be used as possible storage states of vacuum-UV energy above the ionization threshold, those observed in the predissociation can be used as good intermediate states for double resonance experiment, since they are mixtures of different electronic states with long lifetimes.

According to the Fano's multi-level formula for the configuration interaction, the absorption cross section vanishes once in each of successive resonances. ${ }^{4}$ The experimental dissociation cross section (lower trace in Fig. 2), however, does not show such zero cross sections. Although sharp dips may disappear by the overlap of the rotational bands and the laser bandwidth, broad resonances should not be affected by these broadening effects. This lack of zero cross section can be ascribed to the existence of a noninteracting, or weakly interacting dissociation channel.

Figure 6 shows a vertically expanded ionization spectrum of $\mathrm{Cs}_{2}^{+}$. It is evident that $\mathrm{Cs}_{2}$ has a continuous absorption band near $17050 \mathrm{~cm}^{-1}$ with a band width of $50 \mathrm{~cm}^{-1}$ (fwhm). The most likely candidate for the origin of this broad band is the absorption by the (2) ${ }^{3} \Sigma_{u}^{+}$state. The $D^{1} \Sigma_{u}^{+}$state has no direct coupling to the continuum [(2) $\left.{ }^{3} \Sigma_{u}^{+}\right]$in the first order approximation. The $D^{1} \Sigma_{u}^{+}$state is, however, coupled to the $\Omega=0$ component of the (2) ${ }^{3} \Pi_{u}$ state through the spin-orbit coupling, which, in turn, interacts with the $\Omega=1$ component of the (2) ${ }^{3} \Sigma_{u}^{+}$state through L-uncoupling. Thus the nominal (2) ${ }^{3} \Sigma_{u}^{+}(\Omega=1)$ state has some character of the $D^{1} \Sigma_{u}^{+}$state, from which it borrows additional oscillator strength. Since the (2) ${ }^{3} \Sigma_{u}^{+}(\Omega=1),(2){ }^{3} \Pi_{u}(\Omega=1)$, and $C^{1} \Pi_{u}$ states are not directly coupled to the $D^{1} \Sigma_{u}^{+}$and (2) ${ }^{3} \Pi_{u}(\Omega=0)$ states in the first order, this additional dissociation cross section can be incoherently added to the dissociation cross section contributed by the coupling between the states with $\Omega=1$. This then explains the apparent lack of the zero cross section in the experimental PFY spectrum. At frequencies higher than $17200 \mathrm{~cm}^{-1}$, the (3) ${ }^{3} \Sigma_{u}^{+}$state and its nonadiabatic coupling with the (2) ${ }^{3} \Sigma_{u}^{+}$state need to be included in the consideration of the dissociation cross section.

We showed that interference can occur between the continuum and discrete state as long as the continuum has oscillator strength from the initial state, even when the discrete state does not have the oscillator strength. ${ }^{18}$ This is because the discrete state is modified by an admixture of continuum, the contribution of which to the transition dipole matrix element $\left[\left(\psi_{e}|T| i\right)\right]$ interferes with those of the continuum, as explained by Fano's configuration interaction theory. 


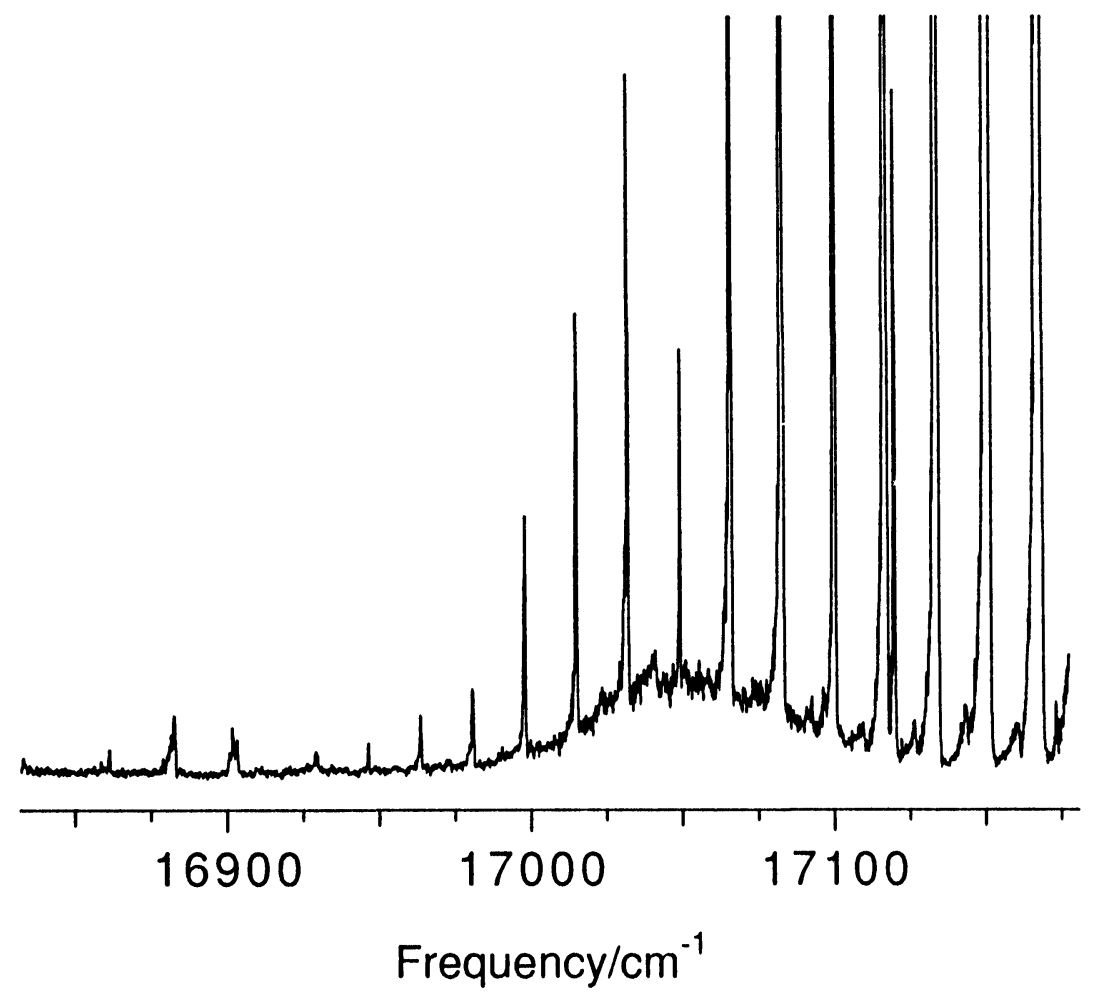

Figure 6 Expanded part of the two photon ionization spectrum of $\mathrm{Cs}_{2}$ shown in Fig. 2. $\mathrm{Cs}_{2}^{+}$ion is monitored. A broad absorption band is seen centered at $17050 \mathrm{~cm}^{-1}$.

The spectrum in Fig. 2 shows that the line shape parameter changes sign at least twice. This kind of the gradual sign change of $q$ is called a 'q-reversal'. While several examples of the q-reversals have been observed in autoionization, they are interpreted as occurring through a structured continuum which is perturbed by another discrete state from the third channel. ${ }^{19}$ Thus, the q-reversal in autoionization is considered as a result of 3-channel interaction. On the other hand, we showed that in predissociation the q-reversal can occur when only 2 channels are involved. ${ }^{18}$ This can be ascribed to the property of the dissociating continuum. While the coupling of the ionization continuum to discrete state shows monotonous variation in autoionization, that of the continuum of nuclear dissociation shows oscillatory variations in the case of outer limb crossing. It is likely that such oscillatory variations have caused q-reversal with only 2 channels involved.

\section{Acknowledgement}

This work was supported by a grant-in-aid for Scientific Research on New Program (05NP0301) by the Ministry of Education, Science, and Culture. 


\section{References}

1. C. Jouvet and B. Soep. J. Chem. Phys., 80, 2229 (1984).

2. R. B. Metz, S. E. Bradforth, and D. M. Neumark, Adv. Chem. Phys., 81, 1 (1992).

3. R. D. Levine and R. B. Bernstein. Molecular Reaction Dynamics and Chemical Reactivity (Oxford University, New York, 1987) Chap. 7.

4. U. Fano. Phys. Rev., 124, 1866 (1961).

5. C. B. Collins, F. W. Lee, J. A. Anderson, P. A. Vicharelli, D. Popescu, and I. Popescu. J. Chem. Phys., 74, 1067 (1981).

6. B. Kim and K. Yoshihara. J. Chem. Phys., 98, 5990 (1993).

7. B. Kim and K. Yoshihara. J. Chem. Phys., 99, 1433 (1993).

8. B. Kim. J. Chem. Phys., 99, 5677 (1993).

9. B. Kim and K. Yoshihara. Chem. Phys. Lett., 202, 437, (1993).

10. B. Kim and K. Yoshihara. Chem. Phys. Lett., 204, 407, (1993)

11. B. Kim and K. Yoshihara. Chem. Phys. Lett., 212, 271, (1993).

12. B. Kim and K. Yoshihara. J. Chem. Phys., 100, 1849 (1994).

13. N. Spiess. Ph. D. thesis, Fachbereich Chemie, Universität Kaiserslautern, 1989.

14. M. Raab, G. Honing, W. Demtröder, and C. R. Vidal. J. Chem. Phys., 76, 4370 (1982).

15. H. Kato, T. Kobayashi, M. Chosa, T. Nakahori, T. Iida, S. Kasahara, and M. Baba. J. Chem. Phys., 94, 2600 (1991); B. Kim, to be published.

16. F. Gounand, T. F. Gallagher, W. Sandner, K. A. Safinya, and R. Kachru. Phys. Rev., A, 27, 1925 (1983).

17. D. Delande, A. Bommier, and J. C. Gay. Phys. Rev. Lett., 66, 141 (1991).

18. B. Kim, K. Yoshihara, and S. Y. Lee. Phys. Rev. Lett., 73424 (1994).

19. A. Giusti-Suzor and H. Lefebvre-Brion. Phys. Rev., A, 30, 3057, (1984). 